\title{
Cupole e facciate loggiate nella architettura chiesastica siciliana del Settecento
}

\author{
Stefano Piazza *
}

\begin{abstract}
SINTESI
II prospetto chiesastico concepito come torre belvedere, legato all'esigenza di fornire alle monache di clausura un luogo da cui poter godere del paesaggio ed assistere a distanzá alla vita cittadina, costituisce una soluzione architettonica discretamente diffusa nei complessi conventuali femminili della Sicilia settecentesca. Si tratta di un tema prettamente siciliano le cui componenti genetiche potrebbero tuttavia, in assenza di riscontri italiani, connettersi ad opere spagnole come la facciata della chiesa de

las Gaitanas a Toledo, il cui periodo di realizzazione (metà del XVII secolo) precede le opere siciliane fin oggi note. II tema della loggia belvedere, nel corso del Settecento, ebbe comunque nell'isola una grande variabilità di forme e applicazioni, conoscendo interessanti ibridazioni con le facciate a tre ordini o, ancora, autonome conformazioni in torri belvedere emergenti dai monasteri. Anche in quest'ultimo caso

sembrano riproporsi possibili confronti con l'architettura spagnola. Alla rielaborazione del belvedere conventuale

\section{ABSTRACT}

The church facade conceived as belvedere tower, linked to the demand of providing to the cloistered nuns a place from which they could enjoy the landscape and to assist from distance to the city life, it represents quitly widespread architectural solution in the female conventual buildings of Sicily of the XVIII century. It deals with a tipically sicilian theme whose genetic components could however, in absence of italian comparisons, connect itself to spanish works like the church of las Gaitanas in Toledo, whose realization period (half XVIII century) goes before the sicilian works knowm till now. The theme of belvedere loggia during the XVIII century had a great variability of shapes and applications in the island, knowing interesting hybridizations with the facades of three orders or besides indipendent conformations in emergent belvedere towers from monasteries. Even in the latter case possible comparisons with spanish architecture seem to arise again. The loggiati which were realized in Palermo, Catania and Caltagirone around the calotte of centric plan, in the second
\end{abstract}

* Facoltà di Architettura. Università degli Studi. Palermo. 
vanno ricondotti i loggiati che nella seconda metà del XVIII secolo si realizzarono a Palermo, a Catania e a Caltagirone intorno alle calotte di chiese a pianta centrica. Opere numericamente esigue, ma architettonicamente pregnanti, che si posero come singolari alternative alla generalizzata tendenza locale a risolvere la volumetria esterna degli impianti centrici enfatizzando il ruolo urbano della facciata. E' grazie anche a queste opere che la cultura siciliana del periodo, impegnata tra l'assimilazione di influssi neoclassici e l'indugiare sul lessico barocco, si dimostra comunque aperta a disinibite sperimetazioni originali. half of the XVIII century, could be lead again to the elaboration of the conventual belvedere. These works are numerically small but architecturally pregnant which impose themeselves as singular alternatives to the local generalized tendency to solve the external volumetry of centric plants emphasizing the urban role of the facade.

Thanks to such works, the sicilian culture of the second half of XVIII century, engaged between the assimilation of neoclassical influences and the delay over the baroque lexicon, shows itself however opened to original disinhibited experimentations.

Negli ultimi decenni del XVII secolo, seguiti alla sconfitta della rivolta messinese nel 1678, Palermo, impostasi come capitale incontrastata dell'isola, conobbe una significativa ripresa dell'attività edificatoria, in cui ebbero un ruolo determinante i rinnovati programmi costruttivi degli ordini religiosi, giunti ormai al culmine della loro ascesa economica e sociale. Nel corso di questa fortunata stagione, la città concluse il suo contributo alla ricerca sugli impianti centrici di ascendenza barocca. I primi impianti a matrice ovale erano stati realizzati negli anni trenta del secolo per le chiese di S. Carlo alla Fieravecchia, legata probabilmente ad un apporto progettuale lombardo, e di S. Mattia ' ${ }^{\prime}$ I due edifici, completati nei decenni successivi, prevedevano come sistema di copertura cupole reali in conci di pietra. Dopo un quarantennio di pausa da queste prima esperienze, nel giro di pochi anni a Palermo si costruirono le chiese di S. Giuliano (intorno al 1679), del SS. Salvatore (dal 1682) e di S. Francesco Saverio (dal 1684), anch'esse completate con cupole reali dominanti la volumetria esterna ${ }^{2}$. Nel corso dei primi decenni del nuovo se-

1 Per le chiese di S. Carlo e S. Mattia cfr. rispettivamente M. R. NobILE, Sicilia-Lombardia 1550-1700. L'architettura, in Lombardi e la Sicilia. Ricerche su architettura e arti minori tra il XVI e il XVIII Secolo, a cura di R. Bossaglia, Pavia 1995, págs. 38-47; ID., II Noviziato dei Crociferi. Misticismo e retorica nella Palermo del Seicento, Palermo 1997. Cfr. anche M. MIRANDA, L. PACE, La chiesa di S. Carlo alla Fieravecchia a Palermo, "Storia Architettura», $n$ ․ 1-2, anno IX gennaio-dicembre 1986, págs. 113-124.

2 Per i riferimenti bibliografici sulle tre chiese palermitane si rimanda ai contributi più recenti: M. Giuffre', Angelo Italia architetto e la chiesa di $S$. Francesco Saverio a Palermo, in L'architettura della Compagnia di Gesu in Italia, XVI-XVIII secolo, Atti del Convegno a cura di L. Patetta e S. Della Torre (Milano, 24-27 ottobre 1990), Genova 1992, págs, 147-153; G. RuBbino, La chiesa di $S$. Giuliano presso Porta Maqueda a Palermo, «Storia architettura», n.s., n.2, Roma 1996, págs. 
colo, tuttavia, l'ormai raggiunta saturazione socio-politica del clero all'interno della città fece registrare un progressivo rallentarsi dell'attività costruttiva religiosa nella capitale dell'Isola, in coincidenza, orientativamente, con l'avvio della grande stagione architettonica della Sicilia sudorientale, seguita al sisma del 1693. La ricerca sugli impianti centrici o centrici allungati, quali l'ovale e l'ottagono irregolare, venne quindi condotta, durante il XVIII secolo, fuori Palermo e in particolare nella Sicilia orientale del dopo terremoto, dove maggiori furono le necessità costruttive e migliori, di conseguenza, le opportunità professionali.

Fondamentale per lo sviluppo della ricerca settecentesca sugli impianti centrici in Sicilia è l'opera di Rosario Gagliardi (1698?-1762), ambiguamente caratterizzata da una palese contraddizione tra i sistemi di copertura adottati nella pratica professionale e quelli legati alla ricerca teorica. L'aspirazione a concludere gli organismi centrici con grandi cupole estradossate, affiorante nella raccolta dei progetti teorici dell'architetto siracusano ${ }^{3}$, sembra infatti scontrarsi con una realtà probabilmente più legata a una logica di economicità e di rapidità di esecuzione dell'opera, ma anche ad un'attenzione per il comportamento antisismico delle coperture, problema non secondario nel contesto della ricostruzione. E' certo comunque che durante il Settecento, contrariamente all'orientamento manifestatosi a Palermo nel secolo precedente, per gli impianti centrici, e in particolare per quelli a matrice ovale, si scelsero prevalentemente volte leggere, in conglomerato o in canne e gesso, incluse in involucri murari coperti a tetto ${ }^{4}$.

137-146; S. Piazza, La chiesa del SS. Salvatore, in E. Di Gristina, E. Palazzotto, S. Piazza, Le chiese di Palermo, Palermo 1998, págs. 107-113.

3 Dei dieci progetti del trattato dedicati a impianti centrici ben nove sono pensati con grandi cupole reali concluse dal lanternino, mentre nella realtà nessuno degli impianti centrici realizzati su progetto di Gagliardi presenta una simile soluzione. Cfr. L. DI BLASI, F. GENOVESI Rosario Gagliardi "architetto dell'ingegnosa citta di Noto», Catania 1972. Sull'intera collezione di disegni attribuibile al Gagliardi cfr. L TRIGILIA, I disegni di Rosario Gagliardi nella collezione Giuseppe Mazza di Siracusa, "ll disegno di architettura», n. 7, aprile 1993, págs. 35-38. Per un approfondimento sulll'opera del Gagliardi si rimanda a: S. TOBRINER, La genesi di Noto, (I ed. 1982) Bari 1989, págs. 138-167; D. Germano', Rosario Gagliardi, architetto siciliano del '700, Roma 1985; ID., Barocco in Sicilia, chiese e conventi di Rosario Gagliardi, Firenze 1986, L. TRIGILIA, I/ corpus di disegni di Rosario Gagliardi. Lo studio dei trattati e l'uso dei modelli nell'attività del maestro, "Annali del Barocco in Sicilia", 1/1994, págs. 63-78; S. BosCArINo, Sicilia barocca. Architettura e città 1610-1760, (I ed.1981) ed. aggiornata Roma 1997, in particolare págs. 166-178; M. R. NoBILE, Piante centriche fra elaborazioni teoriche e prassi costruttive: il contributo di Rosario Gagliardi, in L'architettura del Settecento in Sicilia, a cura di M. Giuffrè, Palermo 1997, págs. 117-123; Si vedano poi i diversi contributi in Rosario Gagliardi e l'architettura barocca in Italia e in Europa, "Annali del Barocco in Sicilia", 3/1996, numero monografico.

4 Sul tema delle volte leggere cfr. M. R. Nobile, Piante ... cit. Vanno comunque segnalate significative eccezioni come la chiesa della Badia di S. Agata a Catania progettata da Giovan 


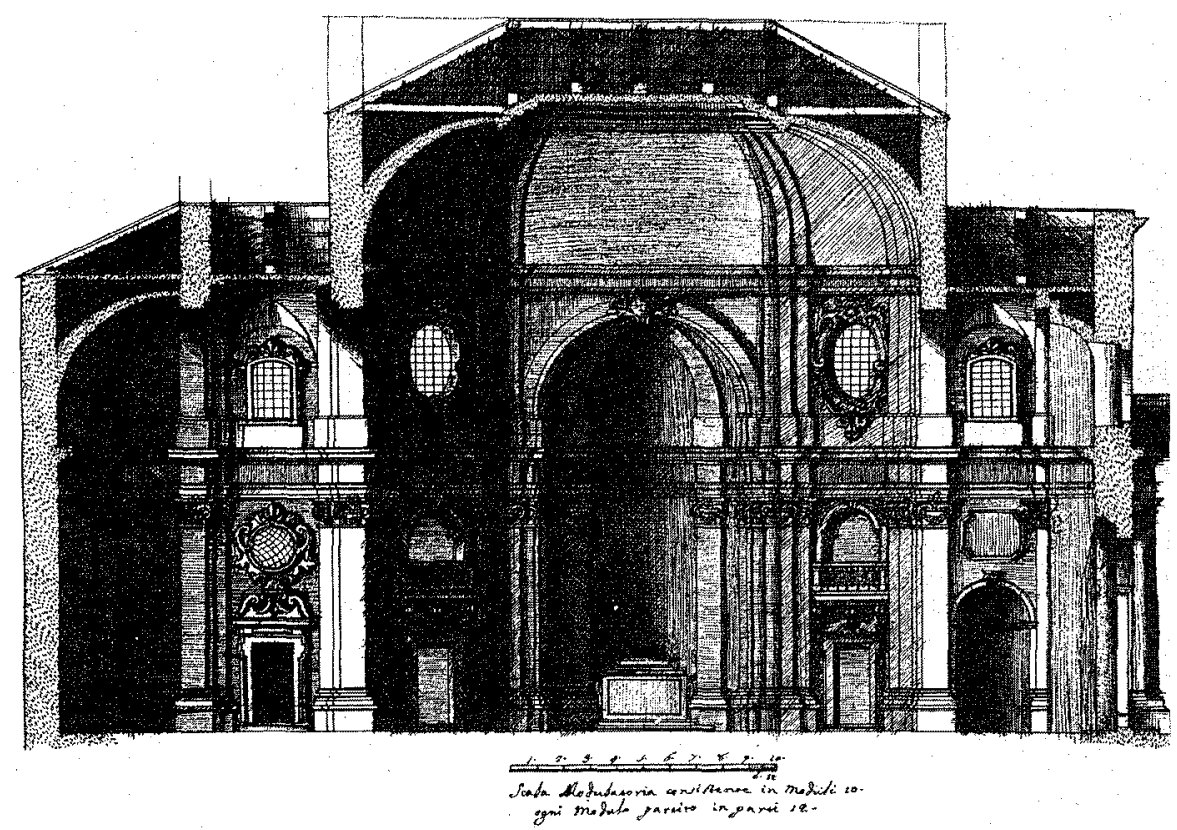

Fig. 1. Sezione longitudinale della "Cnografia D" (Rosario Gagliardi, prima meta del XVIII secolo. Collezione Mazza, Siracusa).

Significativo è il fatto che nella stessa chiesa di S. Carlo alla Fieravecchia a Palermo, in seguito ai danni subiti da un fulmine nel 1727, la cupola reale venne sostituita da una calotta finta, protetta esternamente da un tetto a falde ${ }^{5}$, e che una soluzione analoga era stata proposta - senza essere poi attuata- l'anno precedente per la cupola del SS. Salvatore, gravemente danneggiata da una scossa sismica ${ }^{6}$.

Battista Vaccarini, e quella di S. Giuseppe ad Acicatena, in cui intervenne Francesco Battaglia, entrambe con impianti basati sul cerchio e coperte con grandi cupole reali con lanterna. Volte reali furono realizzate probabilmente anche nella chiesa di S. Antonio a Ferla, anch'essa con un ampio spazio centrale basato sul cerchio, e per gli impianti ovali nella chiesa madre di S. Flavia, vicino Palermo, e in quella del SS. Salvatore a Petralia Soprana. In questi tre casi la grande calotta rimase comunque occultata all'esterno da muri perimetrali.

5 Cfr. M. R. Nobile, Sicilia... cit., pág. 46.

6 Da una relazione del settembre 1726 sappiamo che, prima dell'intervento di consolidamento realizzato da Giacomo Amato e Gaetano Lazzara, una commissione di «ingigneri e architetti » aveva consigliato di «scendere la cubola per allegerirla del peso" cioè di demolire la struttura esistente per crearne una più leggera. Cfr. Archivio di Stato di Palermo, fondo Corporazioni Religiose Soppresse, Monastero del SS. Salvatore, vol.843, ff.116 e sgg. 
Questo tipo di copertura, pur non compromettendo la qualità dello spazio interno, costituiva indubbiamente un problema nell'orchestrazione architettonica esterna dove veniva a mancare l'elemento fondamentale della cupola nella sua doppia funzione di emergenza urbana e nodo conclusivo dell'organismo centrico. Nell'ambito di questa problematica è interessante notare che tra tutti i progetti inseriti nel trattato manoscritto del Gagliardi, l'architetto si mostra indeciso solo in quello della «icnografia D", I'unico dedicato al tema dell'impianto a ottagono allungato con calotta inclusa in un tiburio cieco. Nel disegno di sezione (fig. 1), alla soluzione a basso tiburio con tetto a falde, inglobante la volta, viene sovrapposta quella dove le pareti dell'involucro murario si alzano fino al colmo del tetto. Questa seconda ipotesi progettuale peró e soltanto accennata, assumendo l'aspetto di incerta annotazione ancora da svi-
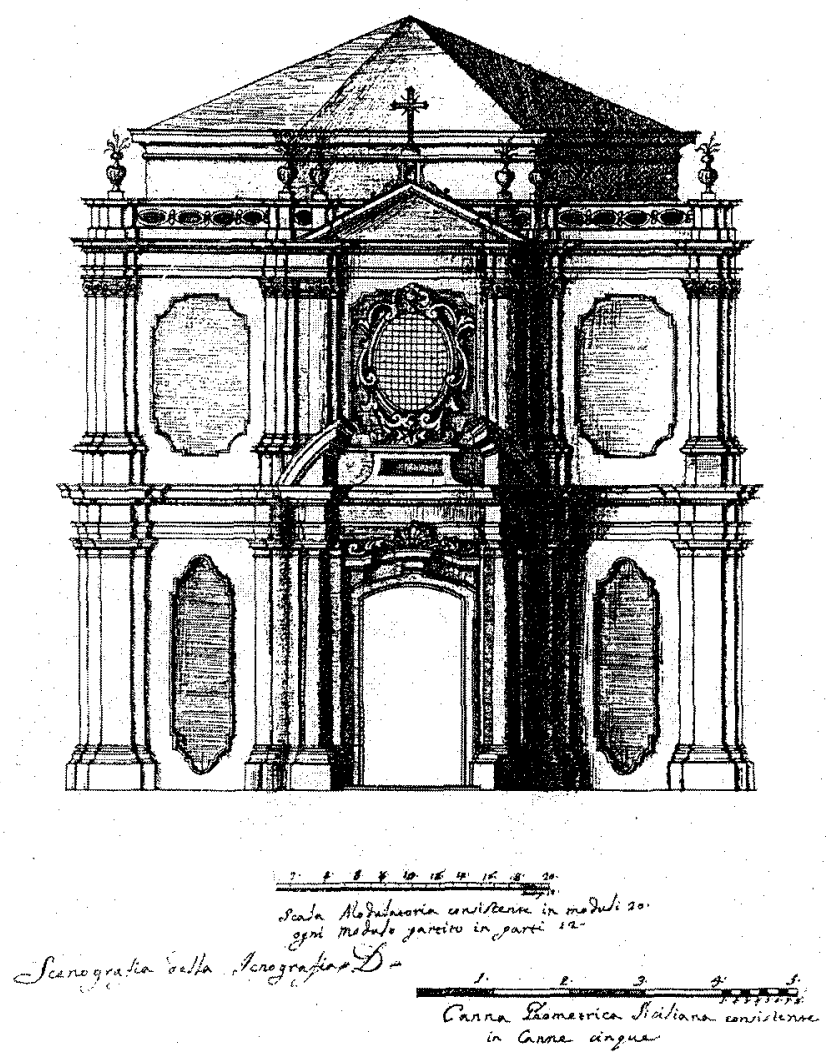

Fig. 2. «Scenografia della icnografia D» (Rosario Gagliardi, prima meta del XVIII secolo. Collezione Mazza, Siracusa). 
luppare ${ }^{7}$. Nel disegno del prospetto (fig. 2) la proiezione frontale del tiburio basso diventa parte integrante del sistema compositivo della facciata, assumendone il ruolo di elemento conclusivo.

Se si guarda l'ampia casistica di impianti centrici e centrici allungati coperti da volte leggere - o in ogni caso da volte occultate all'esterno - che in questo periodo si andavano realizando in Sicilia ${ }^{8}$, è evidente come la prassi costruttiva seguì altre soluzioni.

Al di là delle differenze planimetriche, delle diverse vicende di cantiere e della diversa collocazione cronologica, si riscontra infatti un comune atteggiamento mirato a risolvere la qualificazione volumetrica esterna concentrandosi esclusivamente nella definizione della facciata, e lasciando privi di qualsiasi connotazione architettonica i volumi retrostanti. Un atteggiamento, quindi, concettualmente opposto alla linea di ricerca vittoniana che in quegli stessi anni andava sviluppandosi in Piemonte. Intesa come unico segno urbano dell'edificio, la facciata di queste chiese siciliane, prevalemtemente annesse a monasteri, tende ad imporsi innalzandosi al di là della reale altezza dell'interno per inglobare in sommità le celle campanarie ${ }^{9}$. Un criterio ricorrente, con una grande varietà di soluzioni, nel più ampio panorama dell'ar-

\footnotetext{
7 Per i disegni citati Cfr. L. DI BLASI, F. GENovesLI, op. cit, pp.55, 77-80. Nella più fedele rielaborazione concreta del progetto dell'icnografia $\mathrm{D}$, attuata per la chiesa di S. Chiara a Caltagirone $(1740-48 \mathrm{C}$.), il problema fu lasciato del tutto irrisolto, mostrando con evidenza la debolezza formale di questo sistema di copertura nella orchestrazione esterna dell'edificio. Sulla chiesa cfr. anche $G$. PAGNANO, Modulo e proporzione nei disegni di progetto di Rosario Gagliardi, "Disegnare. Idee-immagini», no 6, 1993, págs. 51-60.

\& Ci riferiamo in particolare alle chiese ovali, annesse a monasteri di benedettine, come: $\mathrm{S}$ Giovanni a Scicli (seconda metà del Settecento); S. Giuseppe a Ragusa (seconda metà del Settecento); SS. Trinità a Catania (F. Battaglia, dal 1745); S. Giacomo a Buscemi (seconda metà del Settecento). Le chiese ad impianto centrico allungato del Carmine a Noto $(1750 \mathrm{c}$.); della SS. Madonna Addolorata e della Madonna del Bosco, entrambe a Niscemi; o l'articolato organismo della chiesa parrocchiale di S. Antonio a Ferla. Si vedano anche gli impianti piú propriamente centrici come le chiese di S. Anna a Piazza Armerina (del 1745) e di S. Giuseppe a Caltagirone. Per le chiese citate si rimanda a: F. MINISSI, Aspetti dell'architettura religiosa del Settecento in Sicilia, Roma 1958; S. BOSCARINO, op. cit.; D. Germanò, Barocco ... cit., P. NIFOSI', Scicli, una via tardobarocca, Modica 1988, págs. 14-24. Per le chiese a pianta ovale si veda M. R. NoBILE, La pianta ovale in Italia (XVI-XVIII secolo). Problematicità del tipo, Tesi di Dottorato, Politecnico di Torino, tutor prof L. Patetta, IV ciclo; ID., Prassi tipologica nella Sicilia del XVIII secolo: le chiese a pianta ovale del Val di Noto, "Annali del barocco in Sicilia", 1/1994, págs. 55-61.

9 Per un orientamento sui caratteri ricorrenti nei prospetti dalle chiese siciliane del Settecento si rimando a S. BOSCARINO, op cit., págs 95-208. Riguardo all'inserimento dei campanili o delle celle campanarie nelle facciate di impianti centrici si confrontino, a titolo orientativo, lo schema a due campanili emergenti dalla sommità del secondo ordine in SS. Trinità a Catania e al suo parallelo nella Sicilia occidentale della chiesa di S. Veneranda a Mazara; quello dei campanili inseriti nel secondo ordine di S. Giuseppe a Caltegirone e S. Antonio a Ferla; e quello a tre livelli con loggia campanaria in sommità nella chiesa del Carmine a Noto e di S. Giovanni a Scicli e di S. Giuseppe a Ragusa.
} 


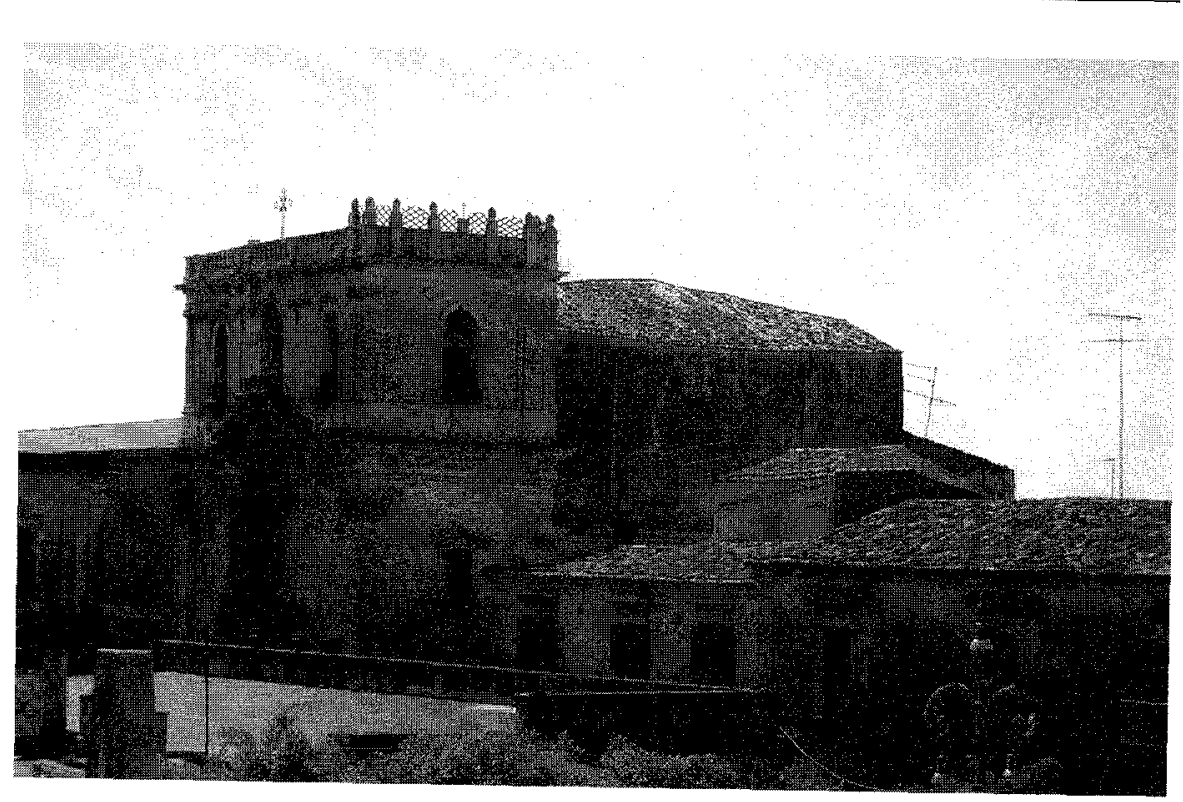

Fig. 3 Noto. Chiesa di S. Chiara, veduta dal piano della cattedrale.

chitettura chiesastica del Settecento siciliano che trova, come è noto, nel tema della «facciata-torre campanaria» uno dei suoi caratteri distintivi. Nei casi più significativi la facciata perde le sue connotazioni di schermo bidimensionale, assumendo l'aspetto di un volume concluso in sé e dotato di una certa indipendenza dal resto del corpo dell'edificio. Su questo tema insiste l'originale soluzione adottata dal Gagliardi per la chiesa di S. Chiara a Noto $\left(1730\right.$ c.) ${ }^{10}$, ad impianto ovale, realizzata per un convento di benedettine. Qui l'aspetto turriforme viene reso con assoluta decisione formale e in rapporto a una chiara motivazione funzionale. II volume a base rettangolare del prospetto è destinato infatti a due distinti spazi di raccolta delle monache: il coro sull'ingresso, aperto verso l'aula $e$, in sommità, un'ampia loggia belvedere, utilizzata anche come cella campanaria (figg. 3,11 ).

II prospetto chiesastico concepito come torre belvedere, legato all'esigenza di fornire alle monache di clausura un luogo da cui poter godere del paesaggio ed assistere a distanza alla vita cittadina, si connette a una piú ampia casistica di strutture loggiate realizzate sulle facciate delle chiese conventua-

10 Sulla chiesa di S. Chiara a Noto cfr. L. Di BIASI, F. GENOVESI, op. cit, pág. 91-96; S. TOBriner, op. cit., págs. 155-162; D. Germano', Barocco... cit., págs. 144-151; págs. 51-60. M. R. Nobile, Le chiese... cit. . Cfr. anche S. Boscarino, op. cit., págs. 167-168. 
li dell'isola. Un tema tutto siciliano, come e stato sottolineato ${ }^{11}$, che manca fin oggi di studi specifici. Gli scarsi approfondimenti conoscitivi sulle singole opere rendono ancora problematica la valutazione della reale diffusione del fenomeno e una sua più precisa collocazione temporale. Difficile risulta quindi il tentativo di rintracciarne le componenti genetiche, in considerazione anche della mancanza di riscontri nel resto d'ttalia. In questa direzione piú illuminante può rivelarsi un confronto con la Spagna, giustificato sia dai noti legami politico-culturali, sia dalla presenza in terra spagnola di opere come la chiesa de las Gaitanas a Toledo (fig. 4), da ricondurre alla meta del XVII secolo, dove la facciata presenta già pienamente sviluppato il tema della loggia ${ }^{12}$.

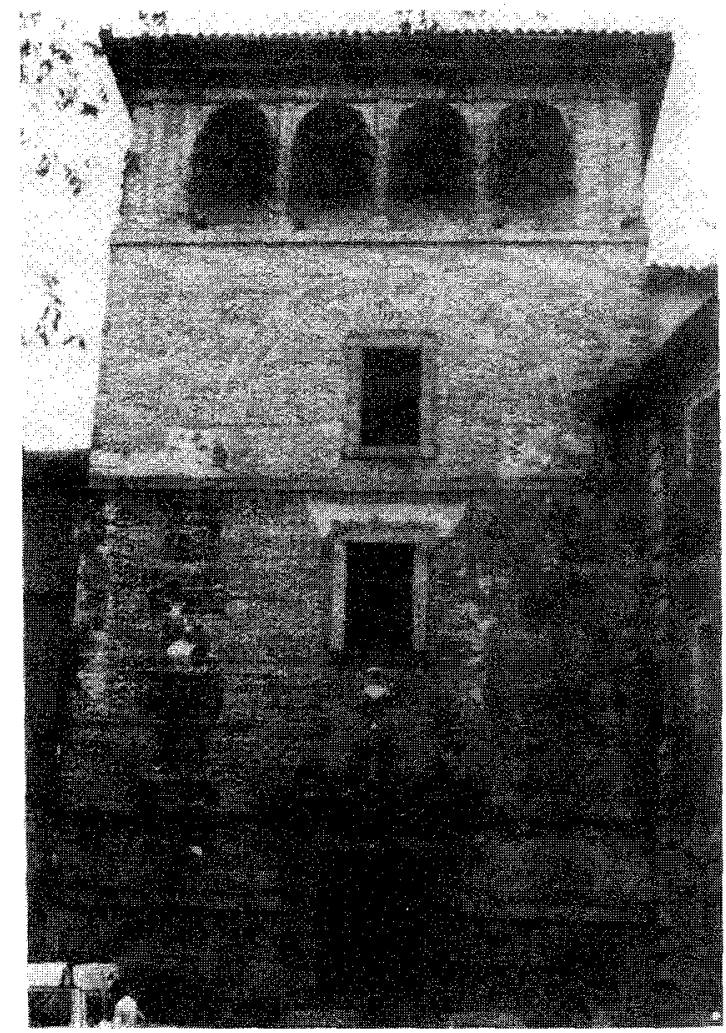

Fig. 4 Toledo. Chiesa de la Gaitanas, prospetto.

1 Cfr. A. M. MAtTEuccl, l'architettura del Settecento, Torino 1988, págs. 175-177.

12 Per la storia del cantiere cfr. D.S. Quevedo, Arquitectura barroca en Toledo: siglo xVII, Toledo 1988, págs. 171-185. 
Allo stato attuale degli studi non è chiaro dove e quando questa soluzione abbia fatto la sua apparizione in Sicilia è non e possibile quindi valutare i rapporti con l'esempio spagnolo. Ma al di là dei possibili contatti con la Spagna è tuttavia indubbio che il motivo ebbe in Sicilia uno sviluppo autonomo con significativi esiti architettonici, tanto da assumere connotazioni endemiche.

Per quanto sia plausibile ipotizzare che le logge in facciata iniziassero a diffondersi in Sicilia nel corso del Seicento, è certo comunque che le maggiori testimonianze di questa tipologia, ancora oggi esistenti, appartengano al XVIII secolo. A Palermo ne rimangono due, entrambe realizzate su precedenti impianti seicenteschi: quella per la chiesa della Badia Nuova (già in
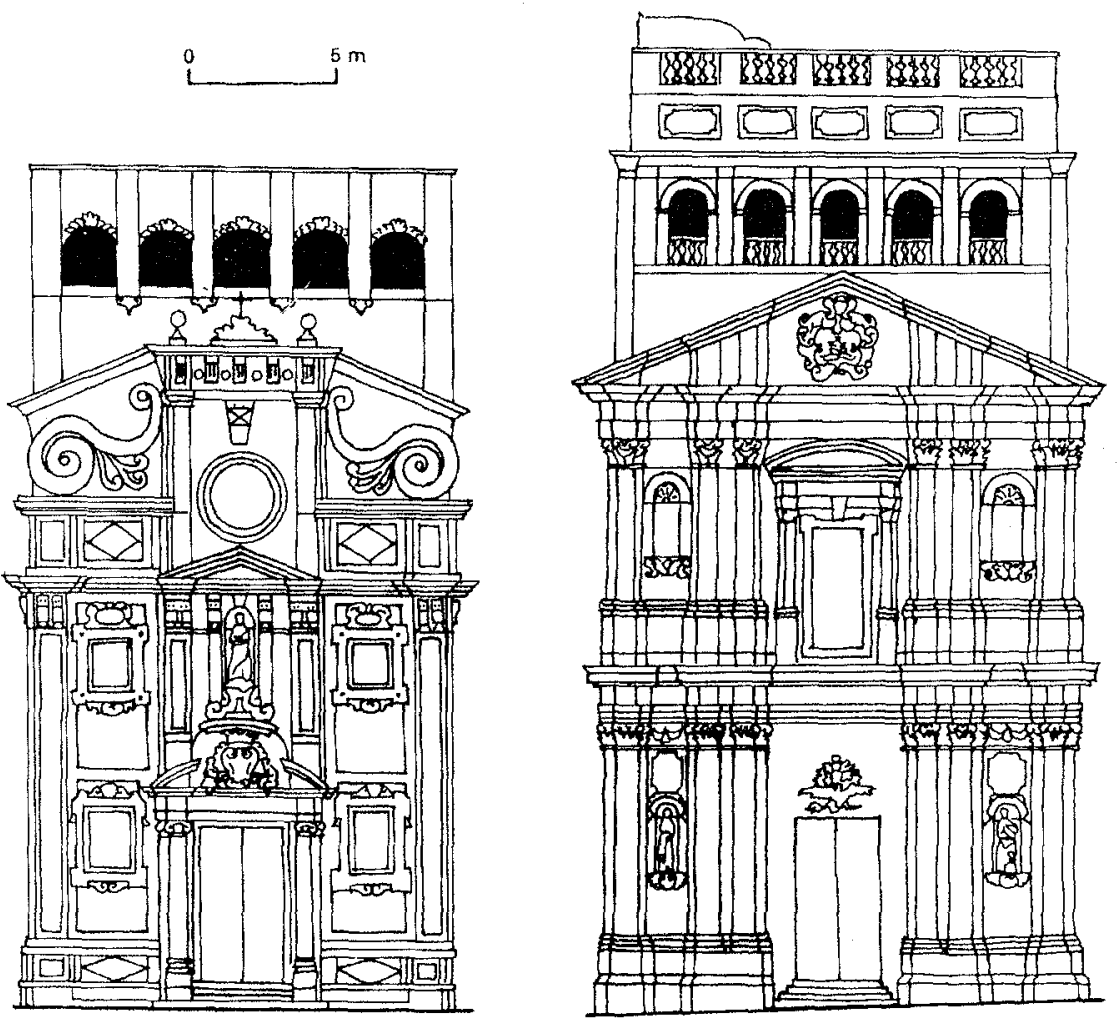

Fig. 5 Palermo. Prospetti della chiesa della Badia Nuova e della chiesa di S. Maria di Montevergini. 
costruzione nel 1730) e quella per la chiesa di Montevergini ${ }^{13}$, realizzata a partire dal 1769 (fig. 5).

Una delle più monumentali della Sicilia occidentale, quella per la chiesa di S. Maria dell'Itria a Sciacca (fig. 6), fu realizzata intorno al 1754 su progetto dell'architetto trapanese Gaspare Gambino, così come certamente

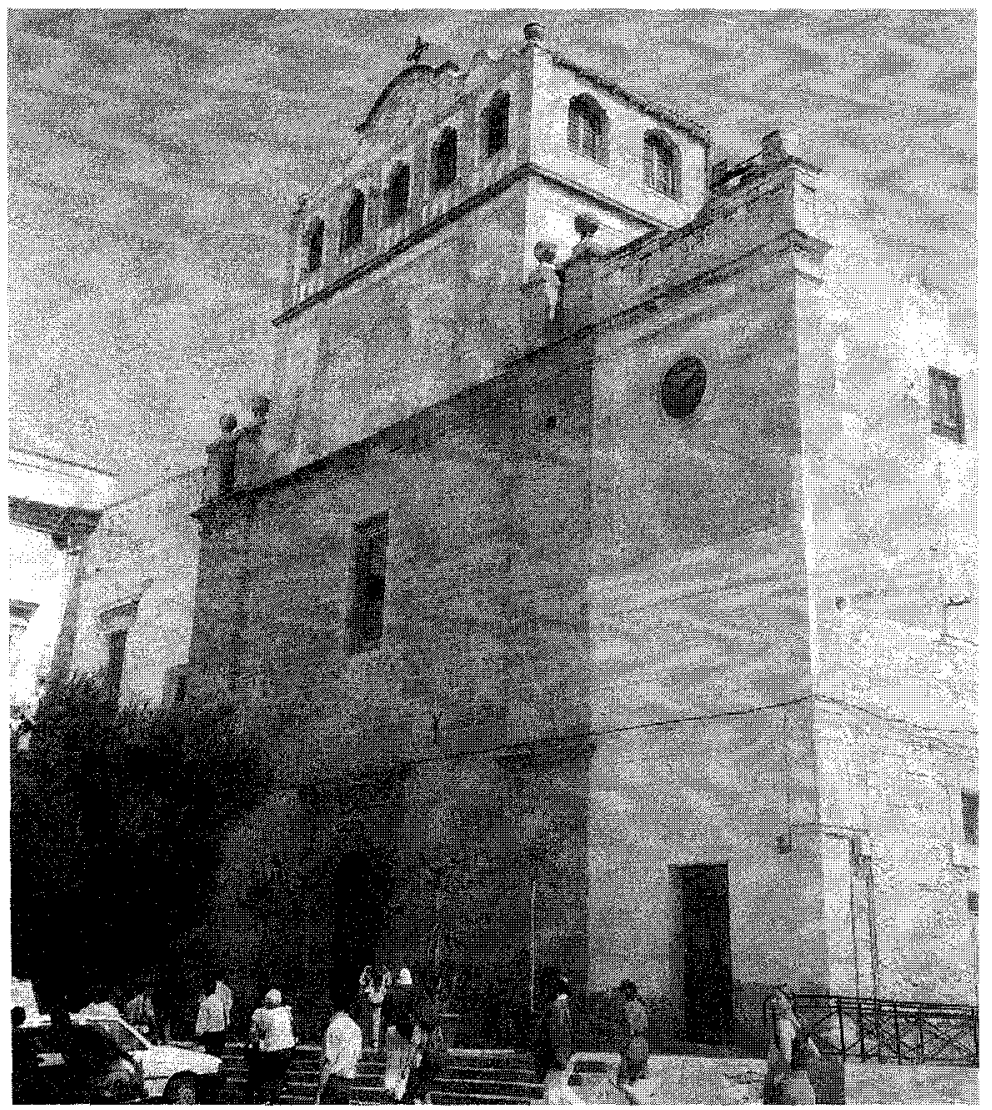

Fig. 6 Sciacca. Chiesa di S. Maria dell'tria, prospetto.

13 Per le due chiese palermitane cfr. G. CARDAMONE, Un cantiere palermitano dell'eta barocca: la chiesa di S. Maria di Montevergini, Palermo 1991; E. NEIL, Architecture in Context: The Villas of Bagheria, Sicily, Ph. D. thesis, Harward Universitiy, Cambridge, Massechussetts, September 1995, págs. 417.Cfr. anche E. DI GristinA, E. PAlazzoto, S. PIAZZA, op. cit. Una terza loggia belvedere, di cui non si conosce la cronologia, fu costruita per la chiesa dell'Immacolata Concezione al Capo. Una sua immagine prima della demolizione si trova in R. LA DuCA, Dalla lanterna al lampione, Palermo 1978, fig. 63. 
da ricondurre al Settecento inoltrato è la facciata della chiesa del conservatorio dalle Verginelle a Catania ${ }^{14}$.

II tema della loggia belvedere ebbe poi nel corso del secolo una grande variabilità di forme e applicazioni, conoscendo interessanti ibridazioni con le facciate a tre ordini (S. Giovanni a Scicli, S. Maria del Carmelo a Noto), o ancora autonome comformazioni in torri belvedere emergenti dai monasteri, come quelle del SS. Salvatore a Noto o della Badia di S. Agata a Catania ${ }^{15}$. Quest'ultimo filone trova una precoce quanto monumentale anticipazione nella torre a doppia loggia del monastero di S. Pietro a Marsala, da ricondurre al tardo Cinquecento, che sembra potersi riconnettere, nell'impostazione volumetrica, alle torri angolari della coeva architettura spagnola.

Alla rielaborazione del tema del belvedere conventuale vanno ricondotti i loggiati che, nella seconda metà del secolo, si realizzarono a Palermo, a Catania e a Caltagirone, intorno alle calotte finte realizzate su chiese a pianta centrica. Queste strutture si posero come elementi di qualificazione dell'involucro murario contenente le volte, in modo da far riemergere il nucleo centrale dell'edificio nei riguardi della quinta in facciata e della scena urbana. In tal modo veniva data un'alternativa alla diffusa tendenza a lasciare non risolta la volumetria esterna contenente le volte a favore della facciata.

Nella chiesa di S. Giuliano a Catania (figg. 7, 11), attribuita a Giovan Battista Vaccarini - anche se resta da chiarire il ruolo di Giuseppe Domenico Palazzotto responsabile del completamento della fabbrica ${ }^{16}$ - il sistema di copertura è costituito da una volta a padiglione reale. La calotta esternamente è circondata da un corridoio anulare loggiato impostato

14. Tra gli altri esempi ricordiamo la chiesa del Collegio di Maria a Geraci Siculo (intorno al 1772) e la chiesa di S. Caterina a Sciacca (fine del XVIII secolo). L'unico esempio fin oggi noto che potrebbe risalire al Seicento è quello della chiesa di $S$. Benedetto a Caccamo, costruita per un monastero di benedettine a partire dal 1615 ma modificata nel XVIII secolo.

15 Per il monastero del SS. Salvatore a Noto(1706-1791) si rimanda a S. ToBRINER, op. cit., págs. 166-167. Cfr. anche S. BoscaRino, op. cit, págs. 182-183, dove viene ipotizzato un interventoe progettuale di Francesco Maria Sortino.

${ }_{16}$ Un modello della chiesa fu relizzato nel 1732 ma la costruzione venne avviata intorno al 1741. La presenza di GIUSEPPE PALAZZOTTO è documentata dal 1746. Ricordiamo che Vaccarini nel 1747 nomina suo procuratore il Palazzotto e che nel 1750 rientra a Palermo lasciando ancora incompiuta la chiesa. Sarà il Palazzotto a completare la fabbrica negli anni sessanta del secolo, più di dieci anni dopo la partenza del Vaccarini da Catania. Sulla chiesa di S. Giuliano cfr. F. FiCHERA, G.B. Vaccarini e l'architettura del Settecento in Sicilia, Roma 1934; G. Policastro, Catania nel Settecento, Catania 1950, págs. 269-270; G. TAlBı La chiesa di San Giuliano a Catania, «Quaderno dell'Istituto di Architettura e Urbanistica», Università di Catania, n. 13, settembre 1983, págs. 19-39; S. Boscarino, Vaccarini architetto, Palermo 1992; cfr. anche ID, Sicilia ...cit., págs. 151-153; Recuperare Catania, a cura di S. BARBERA, Roma 1997, págs. 193-202. 


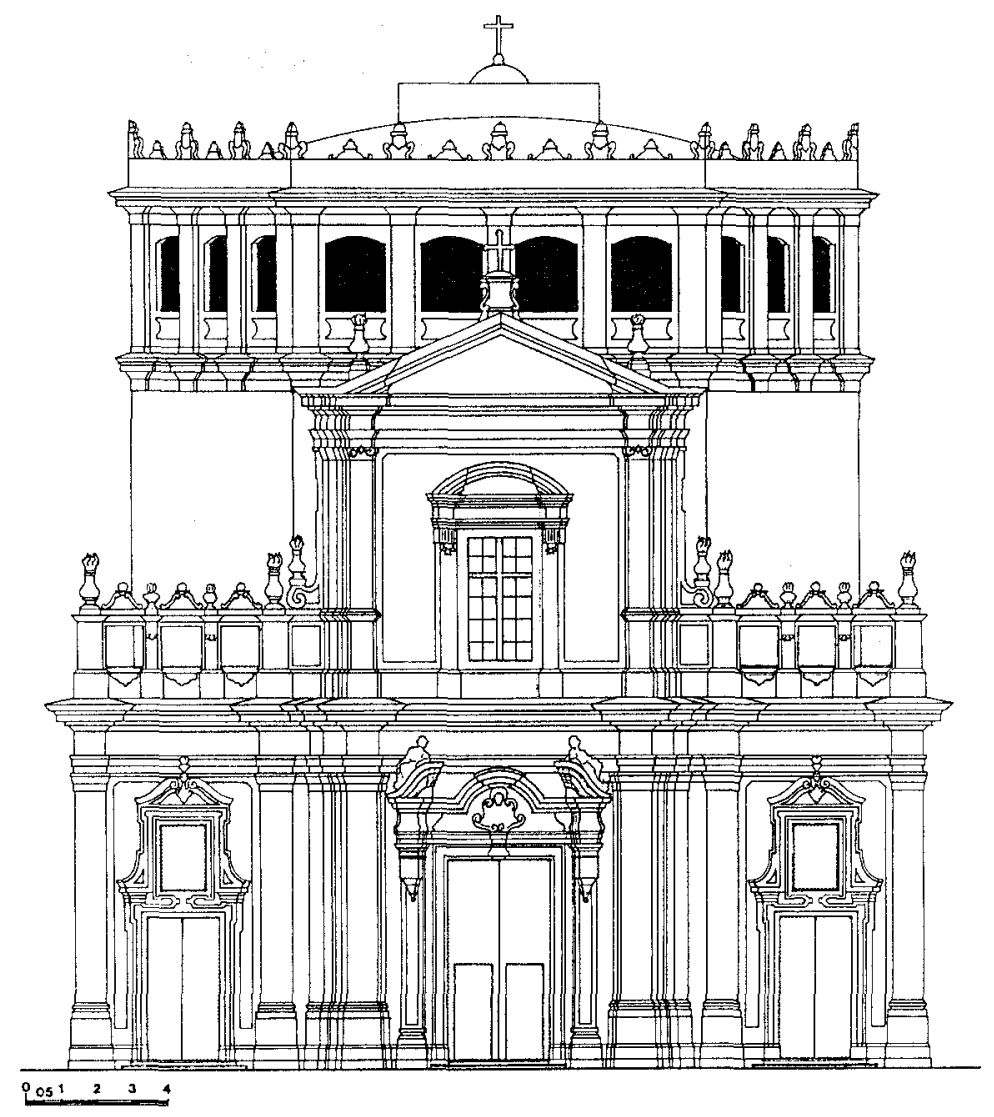

Fig. 7 Catania. Chiesa di S. Giuliano, prospetto su via Crociferi (rielaborazione del rilievo di G. Taibi, 1983).

sui muri d'ambito dello spazio interno della chiesa e svettante al di sopra della facciata, di cui richiama alcuni elementi decorativi nell'originale merlatura di coronamento. Questa inedita emergenza, utilizzata dalle monache benedettine come belvedere, costituisce l'elemento catalizzatore della scena urbana circostante.

La soluzione adottata si pone certamente come un neologismo architettonico siciliano. Nel tentativo di rintracciare fuori dall'Isola possibili componenti di derivazione rischieremmo in questo caso - scavalcando le vicine capitali culturali di Roma e Napoli dove non sembra potersi riscontrare nulla del genere - di imbrigliarci in congetturali riferimenti piú lontani nello spazio e nel tempo quali, per esempio, certi tiburi lombardi. 


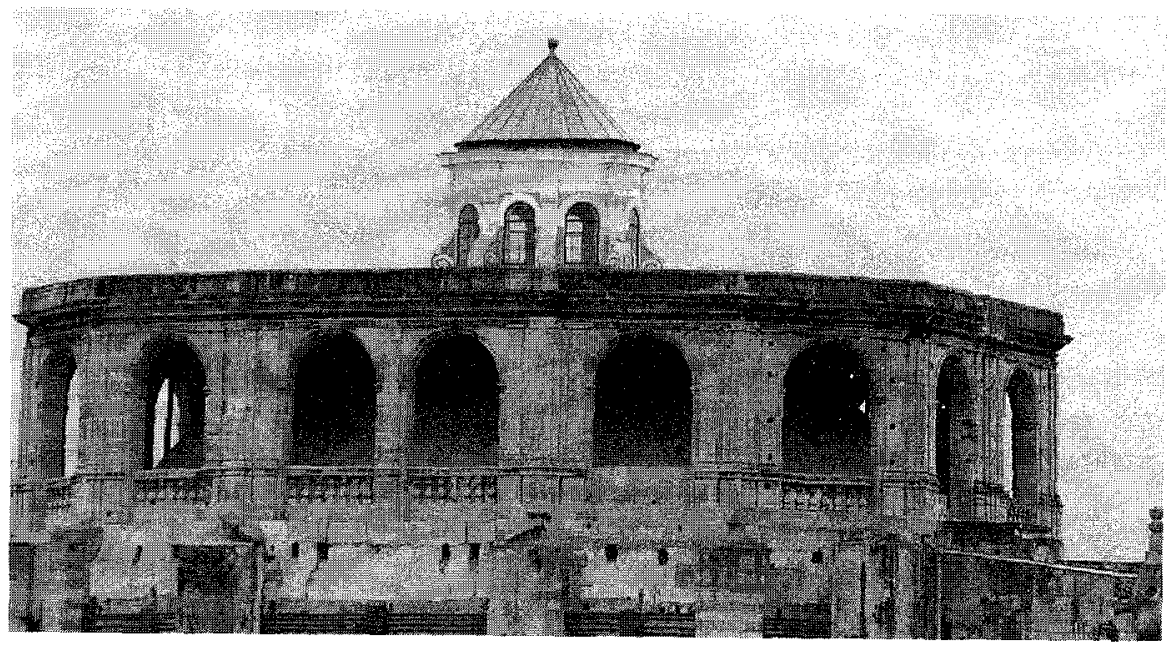

Fig. 8 Palermo. Chiesa del SS. Salvatore, cupola loggiata.

Da un'orazione inaugurale ${ }^{17}$ sappiamo che la chiesa di S. Giuliano, «perfezionata in tutte le sue parti', fu aperta al culto nel 1751 , ma l'opera venne completata solo nella prima metà degli anni sessanta, cioè nello stesso periodo in cui furono costruite la loggia del SS. Salvatore a Palermo (figg. 8,11 ) e, si suppone, quella della chiesa di S. Chiara a Catania, entrambi dotate di coperture loggiate. Le attuali conoscenze quindi non consentono di stabilire se l'idea del loggiato prese forma prima a Catania o a Palermo. Bisogna sottolineare però che la loggia del S. Giuliano è intimamente connessa con il sistema di copertura, e quindi probabilmente era già prevista prima del 1751 , mentre quella del SS. Salvatore a Palermo, unico esempio di cupola loggiata nella capitale dell'Isola, nacque su un edificio già esistente e non venne costruita né per esigenze sociali della committenza, non avendo, almeno nell'idea iniziale, il ruolo di belvedere, né come risultato di una ricerca volta a risolvere il problema della volumetria esterna, in questo caso gia dominata, da settanta anni, dalla più grande cupola ovale della Sicilia. La struttura fu realizzata come risposta a istanze schiettamente pratiche. Da una relazione del 1763 sappiamo infatti che l'idea della loggia fu avanzata da Vincenzo Giovenco, dilettante di architettura, per risolvere definitivamente i problemi di Infiltrazione dell'acqua piovana che da decenni affligevano la cupola ${ }^{18}$. E'

Cfr. P. R. RIzZARI, Orazione inanguratoria per l'apertura della nueva chiesa, Catania 1751.

18 In una relazione del 2 febbraio 1763 Salvatore Attinelli e Domenico Maniscalco, Capomastro della Citta, assistiti da Vincenzo Giovenco, dichiarano intatti che il miglior modo di proteggere la 
interessante notare che il cantiere fu seguito da Giovan Battista Cascione Vaccarini, nipote e collaboratore del già citato Giovan Battista Vaccarini ${ }^{19}$. Non possiamo quindi escludere che le sue competenze tecniche in merito derivassero dalla loggia progettata dallo zio a Catania.

Ma anche se lo scambio dialettico tra Palermo e Catania è ancora tutto da chiarire -in rapporto anche all'attività nelle due città di Giovan Battista Vaccarini- fu certamente l'ambiente catanese a sviluppare l'idea. II tema della copertura loggiata trovò infatti la sua conformazione piú matura nella chiesa di S. Chiara a Catania (figg. 9, 11), annessa a un monastero di monache clarisse, ponendosi in logica continuità con la ricerca avviata dal Gagliardi nella chiesa di S. Chiara a Noto. La piccola chiesa catanese, anch'essa a pianta ovale, venne realizzata tra il 1760 e il 1764 da Giuseppe Domenico Palazzotto che, come abbiamo visto, negli stessi anni era impegnato nel cantiere del S. Giuliano ${ }^{20}$. In S. Chiara a Catania l'architetto coniuga l'idea della torre belvedere e quella della cupola loggiata, in modo da compenetrare l'elemento di facciata con il volume retrostante in un opera coerente e del tutto originale. L'organicità della volumetria esterna della chiesa sembra però essere perseguita e raggiunta solo in senso orizzontale, ciò̀ secondo la direzione anteriore-posteriore, restando irrisolto in senso verticale, cioè nel rapporto tra il volume del primo ordine, elementare nella composizione e severo nella scelta formale, e il soprastante loggiato ben più complesso e decorato, in cui sembra concentrarsi tutta l'attenzione dell'architetto. La frattura visiva tra il partito superiore e quello inferiore,

cupola del SS. Salvatore dalle infiltrazioni d'acqua, in vista anche della realizzazione dell'affresco sull'intradosso, e quello di «farsi all'intorno un portico d'architettura reale e perpendicolare alle fabbriche esteriori del moderno Cubbolo». A.S. Pa., fondo Corporazioni religiose soppresse, Monastero del SS. Salvatore, vol.23, ff. 11-12. La paternità dell'opera ci è rivelata da una relazione dell' 11 marzo 1763 stilata da un gruppo di esperti per giudicare la «costruendonsi gran loggia ed astracone secondo la concepita architettonica idea dell'Ecc.mo Sig. D. Vincenzo Giovenco». Tra i firmatari della relazione ricordiamo: Nicolo Cento "professore di matematica", Giovan Battista Cascione «perito eletto dal Rev.mo Vicario», Francesco Ferrigno «Ingegnero della Città", Mariano Sucameli, architetto. Cfr. G. B. ComandE', Alcuni aspetti del barocco in Palermo dal suo nascere alla fine del sec.XVIII, "Quaderni dell'Istituto di Storia dell'Architettura", Roma 1968, págs. 36-37.

Scarsissime le notizie fin oggi note su Vincenzo Giovenco, citato come "nobile cavaliere". Oltre agli interventi nel SS. Salvatore e nelle citata chiesa di Montevergini, sappiamo che fu nominato amministratore generale di una fabbrica di Vasellame aperta a Palermo nel 1766.

Cír. A. G. Alalmo, La grande officina del Minutolo a Messina e le officine palermitane. Giovenco e Sarzana, “L'illustrazione siciliana”, n.1-12, 1955, págs. 6-7; G. CARDAMONE, op. cit., págs. 89-91.

19 In una nota di pagamento Giovan Battista Cascione «ingignero» riceve 8 once «per diversi visilochi e per la relazione che fece in servitio di tutte le opere". Cfr. S. PIAZZA, il cantiere ... cit.

${ }_{20}$ Sulla chiesa di S. Chiara a Catania ctr. G. POlicastro, op. cit., págs. 273-27S; S. BoscaRino, Sicilia barocca ... cit, pág. 156; G. DATO, La città di Catania, Roma 1983, págs. 62 e 73 . Per un confronto con la chiesa di S. Chiara a Noto cfr. D. GERMANO', Barocco...cit., págs. 144-150. 


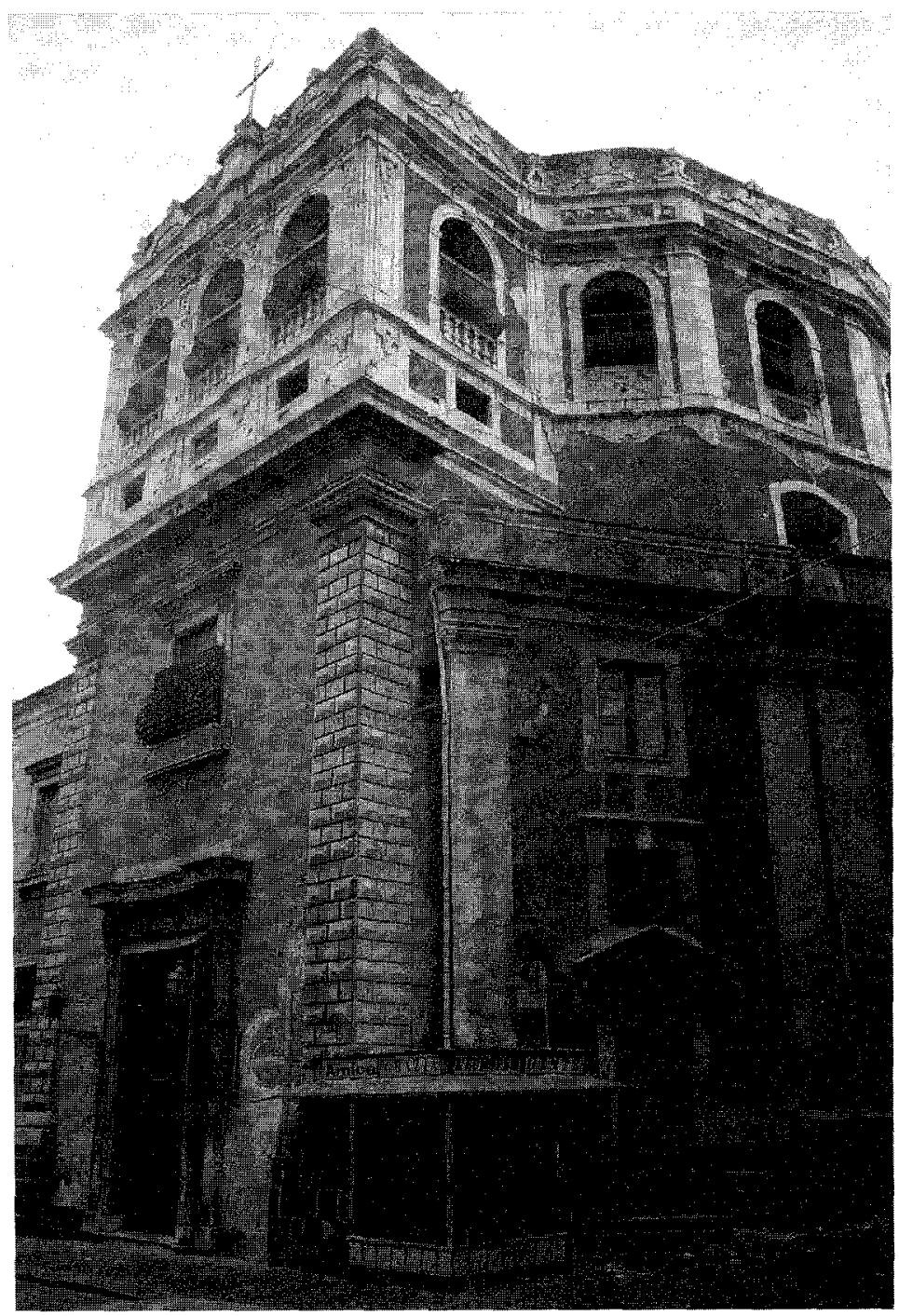

Fig. 9 Catania. Chiesa di S. Chiara, prospetto principale. 
rimarcata dalla sporgenza in facciata di una grossa cornice, alimenta inevitabilmente il sospetto che l' opera sia frutto di due distinti progetti.

A Caltagirone il tema della loggia in copertura si arricchisce di nuove implicazioni. In questa piccola città del Val di Noto, l'operazione condotta nella chiesa di S. Chiara a Catania è ritentata, con esiti meno felici, nella chiesa del S. Salvatore, attribuita al catanese Giovanni Marino ${ }^{21}$, a pianta ottagonale con volta lunettata, realizzata tra il 1759 e il 1794 per un convento di suore benedettine. L'architetto sovrappone al tiburio contenente la volta un loggiato privo di copertura che, rimarcando l'andamento poligonale dell'interno, piega sulla facciata formandone il coronamento. L'effetto però e tutt'altro che felice, tanto che il loggiato sembra un'aggiunta posteriore o comunque il frutto di un ripensamento progettuale a cantiere già avviato. Se infatti nella chiesa di S. Chiara a Catania la loggia si imposta su una facciata predisposta a un tale innesto, nella chiesa di Caltagirone il loggiato deve rapportarsi con un tipo di facciata tradizionale a due ordini di paraste sovrapposte, solitamente concluso da un timpano, nei riguardi

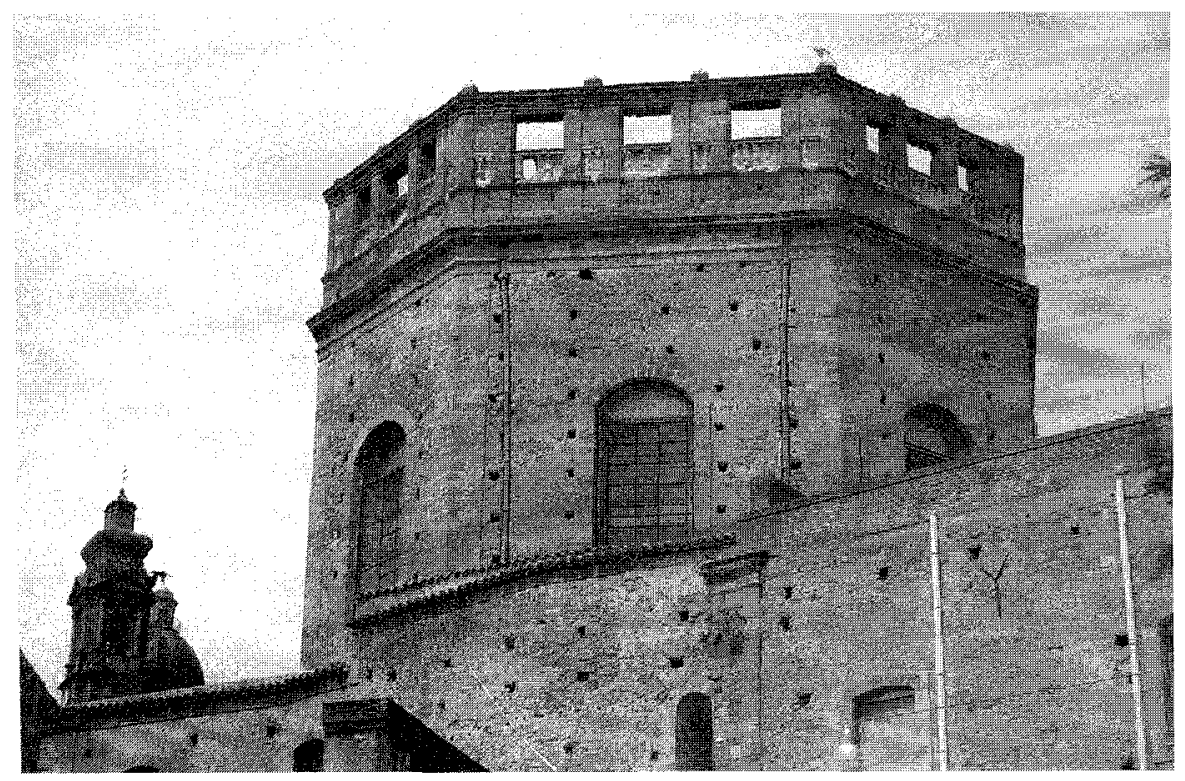

Fig. 10. Caltagirone. Chiesa del S. Salvatore, fronte posteriore.

${ }_{21}$ Sulla chiesa del S. Salvatore a Caltagirone cfr. F. MinisSI, op. cit., págs. 32-35; V. LiBRANDO, La ricostruzione dopo il terremoto del 1693 e L'architettura del Settecento in Caltagirone, Palermo 1977, págs. 176-201; Caltagirone in bianco e nero, a cura di A. Ragona, Caltagirone 1984, págs. 23-24. 

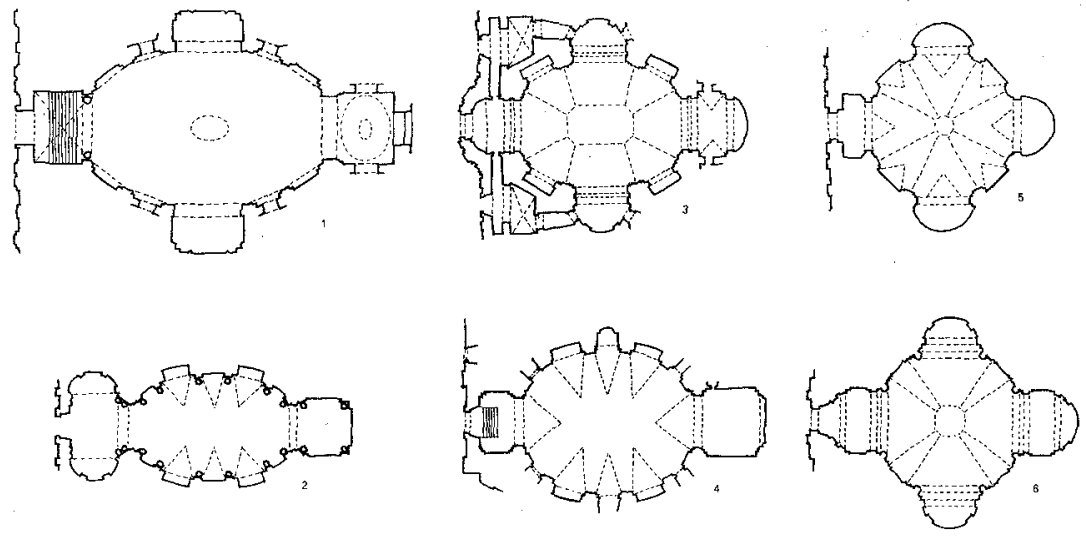

Fig. 11. Quadro sinottico di alcune piante delle chiese citate. 1. Chiesa del SS. Salvatore a Palermo (dal 1682); 2. Chiesa di S. Chiara a Noto (1730 c.); 3. Chiesa di S. Giuliano a Catania (anni quaranta del Settecento); 4. Chiesa di S. Chiara a Catania (1760-64); 5. Chiesa del S. Salvatore a Caltagirone (dal 1759); 6. Chiesa di S. Stefano a Caltagirone (dal 1762).

della quale la loggia si comporta come un corpo estraneo inserito in un sistema compositivo consueto. Nonostante ciò il volume coronato dalla loggia belvedere, se considerato da visuali secondarie (figg. 10-11), acquista una considerevole forza di impatto ambientale, inedita per una piccola chiesa conventuale, riecheggiando con la sua possanza muraria l'immagine di una torre di difesa. L'idea della torre domina l'intera impostazione volumetrica nella chiesa di S. Stefano (fig. 11), sempre a Caltagirone, il cui impianto a ottagono irregolare, con coro sopra l'ingresso e volta a padiglione, presenta notevoli affinità con la vicina chiesa del S. Salvatore. Annessa a un convento di suore francescane, la chiesa, iniziata nel 1762 ma portata a termine tra il 1782 e il 1788, è attribuita da Franco Minissi allo stesso Giovanni Marino, ma sia Antonino Ragona che Vito Librando, pur mantenendo riserve sull'attribuzione del loggiato, la riconducono a un progetto di Francesco Battaglia ${ }^{22}$, anch'esso proveniente da Catania. In ogni caso quindi anche questa opera sembra potersi attribuire a un architetto catanese. La centralità dell'impianto, associata all'idea della loggia belvedere, sembra suggerire per la piccola chiesa francescana un'impostazione turriforme dell'intera configurazione esterna, riducendo a tal fine lo stesso svi-

22 Sulla chiesa di S. Stefano cfr. F. Minissi, op. cit., págs. 40-43; V. LiBRANoo, op. cit, 
luppo della facciata, lasciata poi incompiuta. Nonostante le sue modeste dimensioni l'edificio, grazie al suo sviluppo verticale, riesce così ad imporsi sull'ambiente urbano circostante. La loggia in questo caso ha solo otto aperture architravate poste in alternanza a pannelli maiolicati simulanti altrettante finestre ${ }^{23}$ ed e sovrastata, senza particolari accorgimenti architettonici da un ampio tetto a padiglione.

Ponendo a confronto le diverse logge fin qui esaminate appare evidente come ognuna di esse abbia mantenuto un consistente grado di libertà rispetto alle altre, sia nel rapporto con il resto dell'edificio, sia nell'uso degli elementi costituenti il sistema di copertura. Non è possibile tuttavia dissociare del tutto le singole esperienze progettuali se si considera le inequivocabili affinità tematiche, l'arco di tempo relativamente breve entro il quale furono realizzate e la vicinanza geografica e culturale riscontrabile almeno per le opere di Catania e Caltagirone. II sistema della cupola loggiata, a differenza di quello della loggia in facciata, ebbe uno sviluppo assai limitato- in rapporto al numero di edifici tipologicamente predisposti ad una tale soluzione che non l'adottarono- ma è grazie anche a queste, pur marginali, manifestazioni architettoniche che la cultura locale della seconda metà del Settecento, impegnata tra l'assimilazione di influssi rigoristi neoclassici e l'indugiare sul lessico barocco, si riconferma sempre disposta a nuove, disinibite sperimentazioni, condotte in alcuni casi fuori dal tracciato del linguaggio colto ma in ogni caso vitali e originali. Ed è proprio come pregnante fenomeno locale, e non quindi riproposizione di apporti lessicali e culturali esterni, che lo studio sulle cupole e le facciate loggiate merita di essere sviluppato, attraverso più approfondite ricerche miranti ad individuare gli autori ancora sconoscioti, a disporre secondo una corretta cronologia le diverso realizzazioni, e verificare i possibili contatti iniziali con le esperienze spagnole.

${ }^{23}$ Tra il 1777 e il 1788 si realizzano «i finestroni della Galleria della nuova Chiesa» e i «mattoni di verrate finte fatte da mastro Vito Blandini». Cfr. V. LiBRANDO, op. cit., págs. 194-195. 